\title{
Non-alcoholic fatty liver disease: when to intervene and with what
}

\author{
Authors: Nwe Ni Than ${ }^{A}$ and Philip N Newsome ${ }^{B}$
}

\begin{abstract}
Non-alcoholic fatty liver disease (NAFLD) is increasingly common due to the rising prevalence of obesity in both children and adults. It is associated with metabolic risk factors such as diabetes mellitus, dyslipidaemia and hypertension, and is associated with increased mortality both from cardiovascular-related and liver-related deaths. Identification of those individuals with significant inflammation and fibrosis is a critical part of the patient pathway. Current treatments for NAFLD include weight loss from lifestyle modification or bariatric surgery, management of underlying metabolic risk factors and for those with end stage liver disease, liver transplantation.
\end{abstract}

\section{Introduction}

Non-alcoholic fatty liver disease (NAFLD), defined as accumulation of fat in the liver in the absence of excessive alcohol consumption, is now the most common cause of liver disease in the western world, affecting up to $30 \%$ of the population. ${ }^{1}$ The prevalence of NAFLD rises further in adults with obesity (57-98\%) and diabetes (69\%) reflecting the strong association with metabolic syndromes. ${ }^{1,2}$ Less common causes include use of total parenteral nutrition, rapid weight loss as in the case of jejunoileal or gastric bypass, endocrine disorders (eg polycystic ovarian syndrome, hypothyroidism or hypopituitarism) and usage of drugs such as corticosteroids, oestrogens, anti-retrovirals and tetracycline. Notably there are marked differences in prevalence of NAFLD across ethnic groups, with Hispanic persons having the highest prevalence, followed by non-Hispanic whites, and then African Americans who have the lowest rate of NAFLD. ${ }^{3}$

NAFLD captures a spectrum of disease ranging from simple steatosis to non-alcoholic steatohepatitis (NASH) and on to fibrosis/cirrhosis with the risk of hepatocellular carcinoma. ${ }^{4}$ Data

Authors: ${ }^{A}$ clinical research fellow/specialist trainee in gastroenterology and hepatology, NIHR Centre for Liver Research and Biomedical Research Unit, University of Birmingham, and Liver Unit, University Hospital Birmingham NHS Foundation Trust, Birmingham, UK; ${ }^{\text {B }}$ professor of hepatology, NIHR Centre for Liver Research and Biomedical Research Unit, University of Birmingham, and Liver Unit, University Hospital Birmingham NHS Foundation Trust, Birmingham, UK thus far suggest that NAFLD progresses to NASH in about $20 \%$ of cases, of which 20-25\% may progress on to more advanced stages of hepatic fibrosis and cirrhosis, although predicting which individual patients will progress to develop advanced fibrosis is challenging. ${ }^{4}$ Factors such as higher BMI/weight gain, older age, male gender and presence of metabolic risk factors are associated with higher/worsening levels of fibrosis in cohort studies and recent work has identified genetic polymorphisms that are associated with a higher risk of developing liver fibrosis.,

\section{Pathogenesis}

Although there have been multiple hypotheses addressing the pathogenesis of NAFLD, describing up to three 'hits' for NASH to develop, there is consensus that a common first hit is the hepatic accumulation of triglycerides and free fatty acids. This leads on to the generation of toxic reactive oxygen species, release of inflammatory cytokines and mitochondrial dysfunction, which then lead to initiation and progression to steatohepatitis and fibrosis. ${ }^{4}$ An allele in palatin-like

\section{Key points}

NAFLD is the commonest cause of abnormal liver function tests, which are often requested as part of routine monitoring for chronic conditions such as diabetes or use of statins

Assessing the presence of liver fibrosis is a key step in the workup of a patient with NAFLD

The absolute values of ALT and AST do not provide a guide to the level of liver fibrosis nor to the severity of NAFLD

Assessment of liver fibrosis can be performed using a combination of readily available algorithms alongside transient elastography

Mainstay of management is lifestyle modification which includes diet, exercise and behaviour modification

There are no licensed therapies specifically for the management of NAFLD/NASH although there are many ongoing trials in the area.

KEYWORDS: Non-alcoholic fatty liver disease, cirrhosis, obesity, insulin resistance, treatment 
phospholipase domain-containing 3 gene (PNPLA3) has been strongly associated with increased hepatic fat levels and hepatic inflammation. ${ }^{7}$ This allele was most common in Hispanics, the group most susceptible to NAFLD and it has been reported that the rs738409 CG allele is the most frequent gene variant and common in individuals with NASH. ${ }^{8}$

\section{When to intervene?}

The majority of patients with NAFLD have simple steatosis with no evidence of NASH or fibrosis, and current data would indicate that this cohort is not at an increased risk of liver disease. ${ }^{5}$

Thus, the challenge is to identify which patients have evidence of NASH/fibrosis so that investigation and treatment can be appropriately targeted. While patients with NAFLD commonly have mildly abnormal liver function tests (LFTs), which is often the way by which they are identified, the absolute elevations of transaminases $/ \gamma$-glutamyl transferase (GGT) are not useful in identifying patients with more advanced disease. ${ }^{9}$ Disease stratification can be undertaken by using one of many available algorithms which commonly utilise an aspartate transaminase (AST)/alanine transaminase (ALT) ratio. ${ }^{10}$ Notably the value of the AST/ALT ratio in identifying patients with liver fibrosis holds true even in the setting of normal transaminases. ${ }^{11,12}$ Such algorithms have an excellent negative predictive value albeit with a poorer positive predictive value, and are therefore often combined with an additional non-invasive assessment of liver fibrosis such as transient elastography. ${ }^{13}$ In cases where there is either diagnostic uncertainty, or where confirmation of advanced disease is useful, then liver biopsy should be requested. A potential algorithm for the investigation of such patients is illustrated in Fig 1. Current and future therapies for NASH are summarised in Table 1.

\section{What to intervene with?}

There are no licensed therapies specifically for the management of NAFLD, and therefore current approaches are based

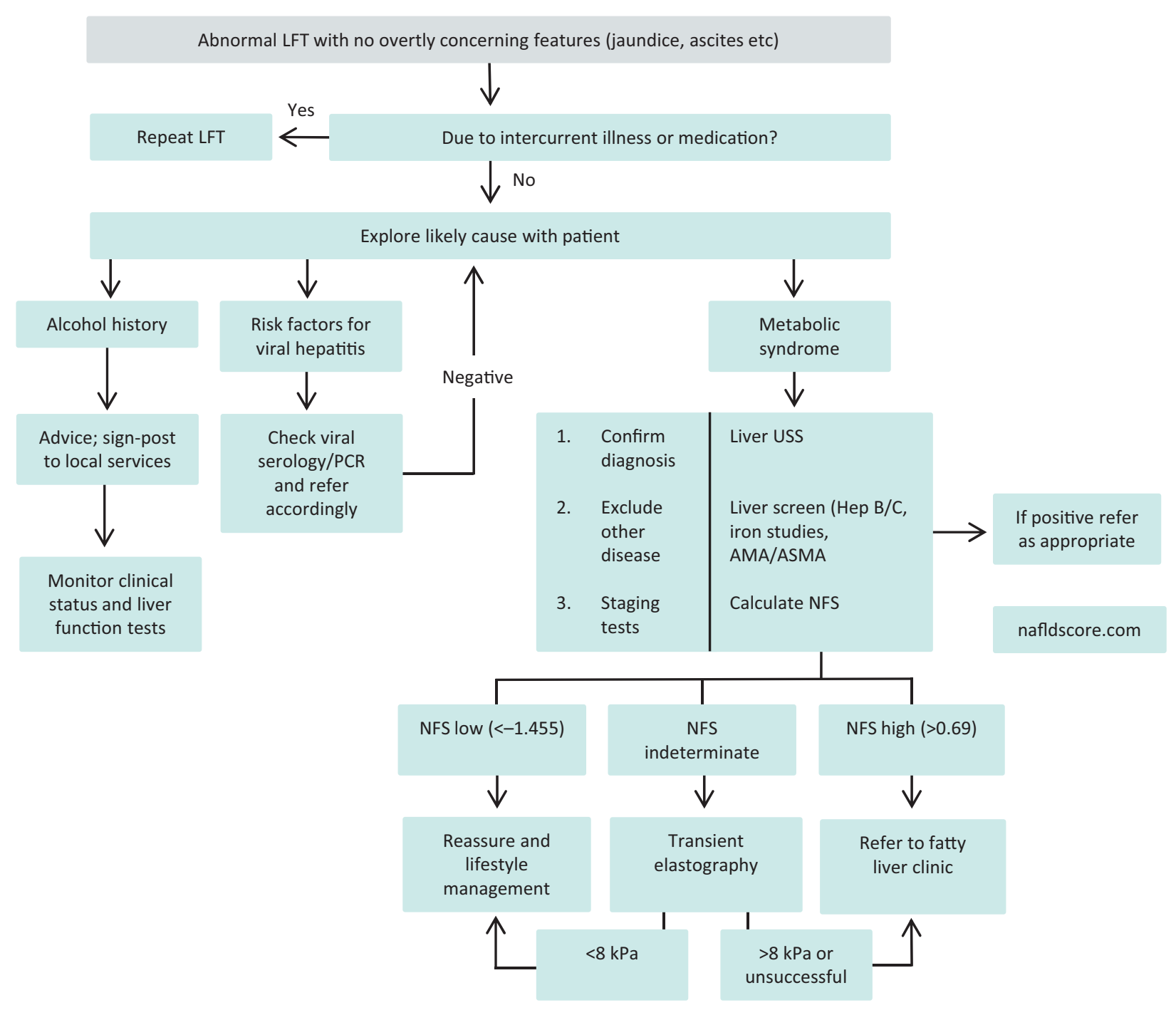

Fig 1. Algorithm for assessing patients with abnormal LFTs. AMA = anti-mitochondrial antibodies; $A S M A=$ anti-smooth muscle antibodies; $L F T=$ liver function test; NAFLD = non-alcoholic fatty liver disease; NFS = NAFLD fibrosis score; PCR = polymerase chain reaction; USS = ultrasound scan. 
Table 1. Summary of current and future therapies for NASH.

\section{Current therapies}

Diet and exercise

Metformin (for patients with diabetes)

Thiazolidinediones (after careful consideration of concomitant pathology such as heart failure, osteoporosis and bladder cancer)

Bariatric surgery (for patients with BMI $>30$ )

Vitamin $\mathrm{E}$ (consider for patients without diabetes)

\section{Future emerging therapies}

GLP-1 receptor agonist eg liraglutide and exenatide

DPP-4 inhibitors (also known as gliptins) eg sitagliptin, linagliptin and saxagliptin

FXR agonists eg obeticholic acid

SGLT2 inhibitors eg dapagliflozin

Galectin 3 inhibitors

DDP-4 = dipeptidyl peptidase-4; FXR = farnesoid X receptor; GLP-1 = glucagon-like peptide-1; NASH = non-alcoholic steatohepatitis; SGLT2 = sodium-glucose co-transporter 2.

on lifestyle management and management of associated comorbidities in anticipation of future pharmacological developments.

\section{Lifestyle modifications}

A reduction in weight of at least $3-5 \%$ has been demonstrated to result in improvements in biochemical indices in patients with NAFLD, while a reduction of at least $10 \%$ is needed to show histological improvements in necroinflammation. A range of different strategies have been utilised with the most effective combining calorific reduction, behavioural modification and exercise, which highlights the importance of behavioural interventions. Such approaches will need to be supplemented by governmental initiatives as outlined by the Academy of Medical Royal Colleges. ${ }^{14}$ As yet, lifestyle interventions have not demonstrably impacted on liver fibrosis although this is likely to require continuation of any measure for a more prolonged period of time than has been undertaken thus far. Notably, exercise without any associated weight loss has also been shown to result in improvements in liver biochemistry and steatosis in NAFLD, which is likely a consequence of improved insulin glucose control, insulin sensitivity and fatty acid oxidation. ${ }^{15}$

Use of pharmacological agents to achieve weight loss in patients with NAFLD has not been particularly successful. Orlistat, a lipase inhibitor, had been demonstrated to improve liver biochemistry and hepatic steatosis in one study, ${ }^{16}$ although a subsequent study revealed no effect on weight nor liver histology. Post-hoc analysis in this latter study revealed that weight loss of $>9 \%$ was associated with histological improvement suggesting that there may be benefit in a time-limited trial of orlistat in some patients. However, given potential issues with fat malabsorption and vitamin $\mathrm{D}$ deficiency, caution should be exercised before commencing orlistat, which should be undertaken in conjunction with expert dietary input.

\section{Pharmacological therapies}

\section{Metformin}

The varied actions of metformin make it an ideal candidate for the treatment of NAFLD; it decreases hepatic gluconeogenesis, increases muscle glucose uptake and enhances fatty acid oxidation in the adipose tissue. However, meta-analysis of studies combining metformin and lifestyle intervention found it did not improve aminotransferases or liver histology, as compared with lifestyle intervention alone. ${ }^{17}$ Notably, this finding was independent of metformin dose or the presence of diabetes. Thus, despite these studies being relatively small in size and likely underpowered, usage of metformin is reserved for those patients with concomitant type 2 diabetes mellitus rather than as a specific treatment for NAFLD.

\section{Thiazolidinediones}

Agents from the thiazolidinedione (TZD) family act principally by enhancing insulin sensitivity through an action on peroxisome proliferator activated receptor- $\gamma$ and increasing circulating adiponectin levels. Smaller studies and a recent meta-analysis indicate that TZDs (mainly pioglitazone) are effective in improving both biochemistry and liver histology in patients with NAFLD ${ }^{17}$ However, in the largest study included in the meta-analysis, the PIVENS trial, pioglitazone did not meet the primary endpoint of histological improvement. ${ }^{18}$ Moreover, there remain concerns about the long-term safety of TZDs with regard to weight gain, osteoporosis, and use in patients with cardiac failure and bladder cancer, which have tempered enthusiasm for their use. Nevertheless, pioglitazone remains a potential therapeutic agent to specifically treat NAFLD in patients, although its long-term usage in this setting remains to be established.

\section{Incretin-based therapies}

Incretins are produced by the intestinal tract in response to food ingestion, where they stimulate glucose-dependent insulin release, decrease glucagon release and prolong gastric emptying, resulting in weight loss, improved insulin sensitivity and glycaemic control. The most common therapeutic agents used are long-acting glucagon-like peptide-1 (GLP-1) analogues, such as exenatide and liraglutide, which have been licensed for the treatment of diabetes mellitus. Studies indicate this class of drugs is safe and potentially effective, with potential in the treatment of NAFLD, ${ }^{19}$ although there are no controlled trials reported with histological outcome data yet.

\section{Cytoprotective and antioxidant agents}

Ursodeoxycholic acid

Ursodeoxycholic acid (UDCA) is a hydrophilic bile acid with cytoprotective and antioxidant properties. UDCA looked encouraging in phase 2 studies, however, when studied in a 
large randomised multicentre trial there was no histological benefit over placebo. ${ }^{20}$

\section{Vitamin E}

The anti-oxidant, vitamin E ( $\alpha$-tocopherol), has been demonstrated to be effective in two large randomised controlled trials (TONIC and PIVENS) in non-diabetic paediatric and adult populations respectively. ${ }^{21-23}$ While both studies showed a significant reduction in histological hepatocellular inflammation and ballooning with vitamin $\mathrm{E}$, there remains uncertainty about their use in diabetic patients, as well as concerns about the safety of doses $>400$ IU/day which have been shown to increase all-cause mortality.

\section{Lipid-lowering agents}

A number of studies have demonstrated that statins can be used safely in the treatment of dyslipidaemia in patients with NASH. However, there are no studies assessing the effect of statins on liver histology, and therefore they cannot be recommended as a specific treatment for NASH. ${ }^{24,25}$

\section{Bariatric surgery}

There are no randomised controlled trials of bariatric surgery in patients with NASH, with published data coming from meta-analyses of cohort series. These series indicate histological improvement in steatosis, lobular inflammation and ballooning, with conflicting outcomes for liver fibrosis. ${ }^{26,27}$ Further studies are required to establish the role and type of bariatric surgery in patients with NASH.

\section{Liver transplantation}

$\mathrm{NASH}$ accounts for an increasing proportion of patients undergoing liver transplantation, despite the associated comorbidities in this cohort. Patient survival is good and, while recurrent steatosis may be common, rates of graft loss due to recurrent disease are minimal. ${ }^{28}$

\section{Conclusion}

With rising obesity levels, the prevalence of NAFLD continues to rise, although the majority of such patients will not develop significant liver disease. For those patients progressing to NASH and advanced fibrosis, intensification of lifestyle management remains the mainstay, while new treatments are tested in clinical trials.

\section{Funding}

NNT and PNT are funded by the National Institute for Health Research.

\section{References}

1 Vernon G, Baranova A, Younossi ZM. Systematic review: the epidemiology and natural history of non-alcoholic fatty liver disease and non-alcoholic steatohepatitis in adults. Aliment Pharmacol Ther 2011;34:274-85.

2 Leite NC, Salles GF, Araujo ALE et al. Prevalence and associated factors of non-alcoholic fatty liver disease in patients with type-2 diabetes mellitus. Liver Int 2009;29:113-9.
3 Kallwitz ER, Kumar M, Aggarwal R et al. Ethnicity and nonalcoholic fatty liver disease in an obesity clinic: the impact of triglycerides. Dig Dis Sci 2008;53:1358-63.

4 Dowman JK, Tomlinson JW, Newsome PN. Pathogenesis of nonalcoholic fatty liver disease. QJM 2010;103:71-83.

5 Ekstedt M, Franzén LE, Mathiesen UL et al. Long-term follow-up of patients with NAFLD and elevated liver enzymes. Hepatology 2006;44:865-73.

6 Anstee QM, Day CP. The genetics of NAFLD. Nat Rev Gastroenterol Hepatol 2013;10:645-55.

7 Romeo S, Kozlitina J, Xing C et al. Genetic variation in PNPLA3 confers susceptibility to nonalcoholic fatty liver disease. Nat Genet 2008;40:1461-5.

8 Sookoian S, Pirola CJ. Meta-analysis of the influence of I148M variant of patatin-like phospholipase domain containing 3 gene (PNPLA3) on the susceptibility and histological severity of nonalcoholic fatty liver disease. Hepatology 2011;53:1883-94.

9 Armstrong MJ, Houlihan DD, Bentham L et al. Presence and severity of non-alcoholic fatty liver disease in a large prospective primary care cohort. J Hepatol 2012;56:234-40.

10 McPherson S, Stewart SF, Henderson E et al. Simple non-invasive fibrosis scoring systems can reliably exclude advanced fibrosis in patients with non-alcoholic fatty liver disease. Gut 2010; 59:1265-9.

11 McPherson S, Anstee QM, Henderson E et al. Are simple noninvasive scoring systems for fibrosis reliable in patients with NAFLD and normal ALT levels? Eur J Gastroenterol Hepatol 2013;25:652-8.

12 Mofrad P, Contos MJ, Haque M et al. Clinical and histologic spectrum of nonalcoholic fatty liver disease associated with normal ALT values. Hepatology 2003;37:1286-92.

13 Petta S, Vanni E, Bugianesi E et al. The combination of liver stiffness measurement and NAFLD fibrosis score improves the noninvasive diagnostic accuracy for severe liver fibrosis in patients with nonalcoholic fatty liver disease. Liver Int 2014, epub ahead of print.

14 Academy of Medical Royal Colleges. Measuring up: the medical profession's prescription for the nation's obesity crisis. London: Academy of Medical Royal Colleges, 2013.

15 Johnson NA, George J. Fitness versus fatness: moving beyond weight loss in nonalcoholic fatty liver disease. Hepatology 2010;52:370-81.

16 Zelber-Sagi S, Kessler A, Brazowsky E et al. A double-blind randomized placebo-controlled trial of orlistat for the treatment of nonalcoholic fatty liver disease. Clin Gastroenterol Hepatol 2006;4:639-44.

17 Musso G, Cassader M, Rosina F, Gambino R. Impact of current treatments on liver disease, glucose metabolism and cardiovascular risk in non-alcoholic fatty liver disease (NAFLD): a systematic review and meta-analysis of randomised trials. Diabetologia 2012;55:885-904.

18 Sanyal AJ, Chalasani N, Kowley KV et al. Pioglitazone, vitamin E, or placebo for nonalcoholic steatohepatitis. $N$ Engl J Med 2010;362:1675-85.

19 Armstrong MJ, Houlihan DD, Rowe IA et al. Safety and efficacy of liraglutide in patients with type 2 diabetes and elevated liver enzymes: individual patient data meta-analysis of the LEAD program. Aliment Pharmacol Ther 2013;37:234-42.

20 Lindor KD, Kowdley KV, Heathcote EJ et al. Ursodeoxycholic acid for treatment of nonalcoholic steatohepatitis: results of a randomized trial. Hepatology 2004;39:770-8.

21 Beaton MD. Current treatment options for nonalcoholic fatty liver disease and nonalcoholic steatohepatitis. Can J Gastroenterol 2012;26:353-7.

22 Lavine JE, Schwimmer JB, Van Natta ML et al. Effect of vitamin E or metformin for treatment of nonalcoholic fatty liver disease in children and adolescents: the TONIC randomized controlled trial. JAMA 2011;305:1659-68. 
23 Chalasani NP, Sanyal AJ, Kowdley KV et al. Pioglitazone versus vitamin $\mathrm{E}$ versus placebo for the treatment of non-diabetic patients with non-alcoholic steatohepatitis: PIVENS trial design. Contemp Clin Trials 2009;30:88-96.

24 Chalasani N. Statins and hepatotoxicity: focus on patients with fatty liver. Hepatology 2005;41:690-5.

25 Lewis JH, Mortensen ME, Zweig S et al. Efficacy and safety of high-dose pravastatin in hypercholesterolemic patients with wellcompensated chronic liver disease: Results of a prospective, randomized, double-blind, placebo-controlled, multicenter trial. Hepatology 2007;46:1453-63.

26 Mummadi RR, Kasturi KS, Chennareddygari S, Sood GK. Effect of bariatric surgery on nonalcoholic fatty liver disease: systematic review and meta-analysis. Clin Gastroenterol Hepatol 2008;6:1396-402.
27 Mathurin P, Hollebecque A, Arnalsteen L et al. Prospective study of the long-term effects of bariatric surgery on liver injury in patients without advanced disease. Gastroenterology 2009;137:532-40.

28 Houlihan DD, Armstrong MJ, Davidov Y et al. Renal function in patients undergoing transplantation for nonalcoholic steatohepatitis cirrhosis: time to reconsider immunosuppression regimens? Liver Transpl 2011;17:1292-8.

Address for correspondence: Prof PN Newsome, Immunity and Infection Department, University of Birmingham, Birmingham B15 2TT, UK.

Email:p.n.newsome@bham.ac.uk
'Interesting, intellectually stimulating. Excellent instructors who are able to bring subjects to life by delivering interactive sessions.' Past MSc student

\section{Applications now open MSc in Medical Education 2015/16}

Delivered by experienced teachers and senior clinical medical education leads and consultants, this part-time, modular programme is designed for doctors wishing to develop in their role as educators. It is designed to equip doctors to use appropriate teaching strategies and educational theories to enhance the way they support learning. Applications close 1 May 2015 